\title{
Often Off-label: Questionable Gabapentinoid Use Noted at Hospital Admission Warrants Deprescribing
}

\author{
Kenneth Lam, MD*; Paula A Rochon, MD, MPH ${ }^{2,3}$; Michael A Steinman, MD4
}

'Department of Medicine, University of Toronto, Toronto, Ontario, Canada; ${ }^{2}$ Department of Medicine and Institute of Health Policy, Management and Evaluation, University of Toronto, Toronto, Ontario, Canada; ${ }^{3}$ Women's College Research Institute, Women's College Hospital, Toronto, Cana$d a ;{ }^{4}$ University of California, San Francisco and the San Francisco VA Medical Center, San Francisco, California.

rat hree years after gabapentin received US Food and Drug Administration (FDA) approval in 1990 for epilepsy, case reports and animal studies emerged announcing its potential in the treatment of pain syndromes through then-novel analgesic mechanisms. ${ }^{1}$ Fast forward 20 years to 2016: gabapentin and its close cousin, pregabalin, are internationally considered first-line agents for the treatment of neuropathic pain in guidelines from the Centers for Disease Control and Prevention, the Canadian Pain Society, and the National Institute for Health and Care Excellence. Gabapentin is the 10th most prescribed drug in the United States, and brand-name pregabalin sales were $\$ 4.4$ billion USD, ranking 8th in invoice drug spending. ${ }^{2}$

The ascendancy of gabapentinoids as drugs of choice for pain, though, is fraught with controversy; yet, they were shepherded to commercial success. In 2004, the patent owner of gabapentin, Warner-Lambert (now owned by Pfizer), admitted guilt to charges that it violated federal regulations in its promotion: they encouraged off-label prescribing through paid physician-to-physician communications, publication of positive outcomes, and suppression of negative ones. ${ }^{3}$ Pfizer paid another settlement in 2009 for false claims about off-label indications for brand-name pregabalin. ${ }^{4}$

Mindful of historical biases, recent trials and meta-analyses have found less favorable outcomes for gabapentinoids in the treatment of off-label pain conditions and greater risks than previously reported. Cochrane reviews for gabapentin demonstrate efficacy only in postherpetic neuralgia (for which it has FDA approval) and diabetic peripheral neuropathy (for which it does not); pregabalin has efficacy in both these conditions as well as posttraumatic neuropathic pain and fibromyalgia (and FDA approval for all four). For other types of neuropathic pain, the evidence is of lower quality. Even for approved indications, the risk-benefit ratio is questionable, as the numbers needed to harm for dizziness and somnolence are similar to the numbers needed to treat for pain. 5,6 Further, case-control studies have found increased odds of opioid-related death when gabapentinoids were coprescribed with opioids, ${ }^{7,8}$ prompting gabapentinoids to be

*Corresponding Author: Kenneth Lam, MD; E-mail: kennethk.lam@mail. utoronto.ca; Telephone: 4163513732 ×2711; Twitter: @drklam.

Received: April 23, 2019; Revised: May 7, 2019; Accepted: May 8, 2019

@ 2019 Society of Hospital Medicine DOI 10.12788/jhm.3245 reclassified as class $\mathrm{C}$ controlled substances in the UK as of April 2019.9

On this backdrop, Gingras and colleagues publish their retrospective cohort study on high-risk prescribing of these popular drugs in Montreal, Canada in this issue of Journal of Hospital Medicine. ${ }^{10}$ In their retrospective cohort study of 4,103 patients admitted to a clinical teaching unit, more than one in eight patients (13\%) were being prescribed a gabapentinoid as an outpatient; chart review of the admission notes indicated that only $17 \%$ of them had an FDA-approved indication and $28 \%$ had no clear indication. Gabapentinoid users were more likely to be coprescribed an opioid than nonusers ( $28 \%$ vs $12 \%$ ). There was no significant difference in length of stay or inpatient death between users and nonusers.

Gingras et al. thereby conclude that there is an opportunity to deprescribe on the basis of few gabapentinoid users having a documented indication and the recent research showing potentials for harm and abuse. ${ }^{11}$ We agree. Messaging around gabapentinoids should be similar to that for opioids: these are medications with limited evidence supporting their use in the treatment of chronic pain, and prescribing them for unapproved indications risks doing greater harm than good. We offer two recommendations on how hospitalists can proceed with deprescribing them safely.

First, the urgency of deprescribing in inpatient settings should be titrated to the degree of risk. When the reason for hospitalization is potentially an adverse drug effect, culprit medications posing a substantial and near-term risk of harm should be stopped, such as when patients on gabapentinoids present with major alteration of their mental status.

In less urgent circumstances, hospitalists should speak first with outpatient prescribers because they may have important contextual information (eg, indication, patient preference, failure of alternative therapies, etc.) about previous care that the inpatient clinician lacks. For gabapentinoids, it is easy to imagine how treated pain syndromes without objective markers of disease may escape notice by a hospitalist and remain undocumented, which may encourage erroneous deprescribing. If the shared decision between the patient and providers is to deprescribe, patients on high doses warrant a tapering schedule. ${ }^{11}$ Pharmacist consultation can help with this.

Second, before discharge, hospitalists should communicate their rationale for deprescribing medications to both 
patients and outpatient prescribers, especially if a prolonged tapering schedule is required. This type of communication occurred infrequently in this study: the reason for deprescribing a gabapentinoid was missing from the discharge summary $55 \%$ of the time. Without this, outpatient prescribers may simply reinitiate the medication after the patient is discharged.

To counter the overuse of gabapentinoids, hospitalists should look for opportunities to deprescribe them where there is concern about adverse events and when evidence-based indications do not exist. Successful deprescribing of these popular drugs will require deliberate collaboration and communication with the outpatient circle of care, as ongoing deprescribing ultimately depends on patients and outpatient prescribers agreeing to the change.

Disclosures: Dr. Steinman served as an unpaid expert witness in United States of America ex. Rel. David Franklin vs. Parke-Davis, Division of Warner-Lambert Company and Pfizer, Inc, litigation which alleged that the named pharmaceutical companies improperly marketed gabapentin for non-FDA-approved uses. Drs. Lam and Rochon have no conflicts of interest to declare.

Funding: Dr. Rochon is supported by the Retired Teachers of Ontario (RTO/ ERO) Chair in Geriatric Medicine at the University of Toronto. Dr. Steinman is supported by the National Institute on Aging, US (K24AG049057 and P30AG044281)

\section{References}

1. Segal AZ, Rordorf G. Gabapentin as a novel treatment for postherpetic neuralgia. Neurology. 1996;46(4):1175-1176. https://doi.org/10.1212/WNL.46.4.1175.

2. Goodman CW, Brett AS. Gabapentin and pregabalin for pain — is increased prescribing a cause for concern? N Engl J Med. 2017;377(5):411-414. https:// doi.org/10.1056/NEJMp1704633.

3. Steinman MA, Bero LA, Chren M-M, Landefeld CS. Narrative review: the promotion of gabapentin: an analysis of internal industry documents. Ann Intern Med. 2006;145(4):284. https://doi.org/10.7326/0003-4819-145-4-200608150-00008.

4. Department of Justice, Office of Public Affairs. Justice Department Announces Largest Health Care Fraud Settlement in Its History. https://www.justice. gov/opa/pr/justice-department-announces-largest-health-care-fraud-settlement-its-history. Published September 2, 2009. Accessed April 12, 2019.

5. Wiffen PJ, Derry S, Bell RF, et al. Gabapentin for chronic neuropathic pain in adults. Cochrane Database Syst Rev. 2017;6:CD007938. https://doi. org/10.1002/14651858.CD007938.pub4.

6. Derry S, Bell RF, Straube S, Wiffen PJ, Aldington D, Moore RA. Pregabalin for neuropathic pain in adults. Cochrane Database Syst Rev. 2019;1:CD007076. https://doi.org/10.1002/14651858.CD007076.pub3.

7. Gomes T, Juurlink DN, Antoniou T, Mamdani MM, Paterson JM, van den Brink W. Gabapentin, opioids, and the risk of opioid-related death: a population-based nested case-control study. PLoS Med. 2017;14(10): e1002396. https://doi.org/10.1371/journal.pmed.1002396.

8. Gomes T, Greaves S, van den Brink W, et al. Pregabalin and the risk for opioid-related death: a nested case-control study. Ann Intern Med. 2018;169(10):732. https://doi.org/10.7326/M18-1136.

9. Mayor S. Pregabalin and gabapentin become controlled drugs to cut deaths from misuse. BMJ. 2018;363:k4364. https://doi.org/10.1136/bmj.k4364.

10. Gingras M-A, Lieu A, Papillon-Ferland L, Lee T, McDonald E. Retrospective cohort study of the prevalence of off-label gabapentinoid prescriptions in hospitalized medical patients. J Hosp Med. 2019;14(9):547-550. https://doi.org/10.12788/jhm.3203.

11. Parsons G. Guide to the management of gabapentinoid misuse. Prescriber. 2018;29(4):25-30. https://doi.org/10.1002/psb.1664. 